УДК 004.896:811.111

Rosetskaya A., Murauyeva A.

\title{
Artificial Intelligence Technology
}

Belarusian National Technical University

Minsk, Belarus

Artificial Intelligence (AI) is a game-changing technology. It has the potential to transform the world. Companies are now significantly making investments in AI to boost their future businesses. Here are some examples of artificial intelligence.

Roxxter Cleaning Robot. Roxxter is a powerful little helper with intelligent navigation software. The robotic vacuum cleaner scans its environment and creates its own map of the entire home. As well as displaying this information to the user in the Home Connect app, the map is also interactive. The RoomSelect function enables the user to select and activate individual rooms on the digital map. If certain areas are not to be cleaned, these can be marked as no-go zones on the map [1]. To achieve the best cleaning results, the motor is located directly on the brush and always provides a strong performance thanks to powerful lithium ion batteries. What's more, this robot has an integrated camera. So, you always know what happens in your apartment or in case you have paranoia about whether you have closed the door or fed the dog, you can relax. And another feature is that Roxxter is currently the only robotic vacuum cleaner that can be started, stopped and even sent to specific rooms via Amazon's cloud-based voice control service, Alexa.

We go further: next robot is for entertainment. It's called Anki's Cozmo. Cozmo is a small, programmable robot with its own personality. It communicates with its eyes, 
movements and sounds. Cozmo can recognize and remember people using a built-in OLED camera: just add in your name and let it stare at you for a few seconds. It'll get excited and say your name back to you in a childlike way. And the next time it sees you, it will recognize you [2]. Users can play with it through its app and interactive cubes that come in the pack. It also allows you to learn programming in a simple and friendly way. In the app, available for Android, IOS and FireOS, you will find some games and skills that Cozmo will help you develop the more you play with it. The app also has a Code Lab that allows you to access the robot's functionality and program it with Scratch.

The next robot will bring more pleasure to your life. It's Emotech Olly - robot with evolving personality. This is the result of the robot's deep-learning capabilities that mean as your Olly gets to know you and your daily routines over time, it will evolve to become more like you and respond to the patterns of your life [3]. Apart is its ability to analyze vocal patterns and adjust accordingly. Olly will offer followup information and even suggestions based on recent interactions you've had with it, and it'll learn to predict behaviors to do stuff like turning on your favorite song each morning as you're getting ready for the day [4].

AI in Biometrics. Biometrics is the measurement and statistical analysis of people's unique physical and behavioral characteristics. The technology is mainly used for identification and access control. There are many types of Biometrics and we'd like to mention some of them. The first two types of biometric identification and recognition solutions are physical and behavioral biometrics. Physical biometric solutions use distinctive and measurable characteristics of particular parts of the human body, such as a person's face, iris, DNA, vein, fingerprints, etc., and transform this information into a code understandable by the 
AI system. Behavioral biometric solutions operate in a similar way, except they use unique behavioral characteristics, such as a person's typing rhythm, way of interaction with devices, gait, voice, etc. [5]. In smartphones, for example, the technology builds a personal profile from the size of a user's fingers, the pressure applied when tapping the screen, where fingers are placed on the screen, the swipe speed, how the device is moved and many other factors.

Fingerprint Recognition. Fingerprint recognition is one of the most well-known biometrics, and it is by far the most used biometric solution for authentication on computerized systems. Most fingerprint biometric solutions look for specific features of a fingerprint, such as the ridge line patterns on the finger, the valleys between the ridges. In order to get a fingerprint match for verification or authorization, biometric systems must find a sufficient number of minutiae patterns. This number varies across systems.

Voice Recognition software gives you the ability to streamline your workflow. Well-designed voice recognition software can help you dramatically increase productivity both at work and at home. You can dictate a document at roughly three times the speed of typing it. And with the right software, you can do so with even more accuracy. What's more, customized voice commands allow for hands-free dictation. That means that you can not only dictate the text to the computer, but say it to open and close a needed file. Even you can send email only by your voice. The great feature of these programs is that they adapt to you. By learning the words and phrases you use the most, the programs get better at dictating your messages over time [6]. With this software, you don't even have to be in front of the computer screen to create documents. Voice recognition apps also eliminate the need for holding a phone. Busy parents who need to send 
emails while using their hands for other tasks can benefit from this software. This software can also be a boon to people with disabilities or injuries that restrict keyboard and mouse use.

A facial recognition biometric system identifies and verifies a person by extracting and comparing selected facial features from a digital image or a video frame to a face database. For example, an algorithm may analyze the distance between the eyes, the width of the nose, etc., and encode the corresponding data as face prints, which can then be used to find appropriate matches in a destination database [5].

References:

1. Mode of access:

https://www.applianceretailer.com.au/2017/09/ifa-2017-boschdebuts-first-robotic-vacuum/. - Date of access: 15.03.2018.

2. Mode of access: https://www.techradar.com/reviews/ankicozmo. - Date of access: 15.03.2018.

3. Mode of access: https://www.cnet.com/products/emotecholly/preview/. - Date of access: 20.03.2018.

4. Mode of access:

https://www.digitaltrends.com/home/emotech-olly-speakerwith-personality/. - Date of access: 22.03.2018.

5. Mode of access: https://www.techemergence.com/ai-inbiometrics-current-business-applications/. - Date of access: 16.03.2018.

6. Mode of access: https://www.business.com/categories/bestvoice-recognition-software/. - Date of access: 15.03.2018. 